\title{
Significant fibrosis after radiation therapy in a patient with Marfan syndrome
}

\author{
Eva M. Suarez, MD, Rebecca J. Knackstedt, BS, Joseph M. Jenrette, MD \\ Department of Radiation Oncology, Medical University of South Carolina, Charleston, SC, USA
}

Marfan syndrome is one of the collagen vascular diseases that theoretically predisposes patients to excessive radiation-induced fibrosis yet there is minimal published literature regarding this clinical scenario. We present a patient with a history of Marfan syndrome requiring radiation for a diagnosis of a right brachial plexus malignant nerve sheath tumor. It has been suggested that plasma transforming growth factor beta 1 (TGF- $\beta 1$ ) can be monitored as a predictor of subsequent fibrosis in this population of high risk patients. We therefore monitored the patient's TGF- $\beta 1$ level during and after treatment. Despite maintaining stable levels of plasma TGF- $\beta 1$, our patient still developed extensive fibrosis resulting in impaired range of motion. Our case reports presents a review of the literature of patients with Marfan syndrome requiring radiation therapy and the limitations of serum markers on predicting long-term toxicity.

Keywords: Marfan syndrome, Radiation therapy, Transforming growth factor beta1, Fibrosis

\section{Introduction}

Marfan syndrome (MFS) is an inherited autosomal dominant trait with an incidence of 1 in 5,000 individuals [1]. Clinical manifestations of the syndrome range from aortic root aneurysm with consequent dissection, long bone overgrowth, and ectopia lentis [2]. The syndrome is associated with mutations of the Fibrillin-1 (FBN-1) proteins [1] which normally acts to regulate transforming growth factor beta (TGF- $\beta$ ) in the extracellular matrix [3]. TGF- $\beta$ is known to play a key role as a mediator of the fibrotic process through effects on fibroblast proliferation, migration, and extracellular synthesis [4]. The significance of TGF- $\beta$ dysregulation resulting in excess fibrosis has been shown in several animal models. When administered exogenously, TGF- $\beta$ initially produces excess granulation tissue that progresses to kidney and liver fibrosis at higher doses [5]. Knock-out mice deficient in FBN1 also demonstrate elevated TGF- $\beta$, as seen in MFS patients, which leads to development abnormalities in the lung [6].

Fibrosis resulting from radiation therapy has been associated with increased levels of TGF- $\beta$ [5]. Out of the three known mammalian isomers of TGF- $\beta$, the development of radiation induced fibrosis is best correlated with TGF- $\beta 1$ [7]. TGF- $\beta 1$ induces collagen gene expression, collagen production, and inhibits collagen degradation $[7,8]$. Therefore, studies have suggested monitoring plasma TGF- $\beta$ or TGF- $\beta 1$ levels in normal patients to predict the risk of developing radiation induced fibrosis [9-11]. Studies thus far have demonstrated a correlation between pre- and post-treatment plasma TGF- $\beta 1$ with the risk of developing radiation-induced pneumonitis [9]. Based on the observations correlating elevated TGF- $\beta$ with fibrosis, MFS patients are thought to be poor candidates

Received 11 March 2014, Revised 18 July 2014, Accepted 29 August 2014.

Correspondence: Eva M. Suarez, MD, Department of Radiation Oncology, Medical University of South Carolina, 169 Ashley Avenue, Charleston, SC 29425, USA. Tel: +1-843-792-7806, Fax: +1-843-792-8898, E-mail: suarezem@musc.edu

(c) This is an Open Access article distributed under the terms of the Creative Commons Attribution Non-Commercial License (http://creativecommons.org/ licenses/by-nc/3.0/) which permits unrestricted non-commercial use, distribution, and reproduction in any medium, provided the original work is properly cited.

www.e-roj.org 


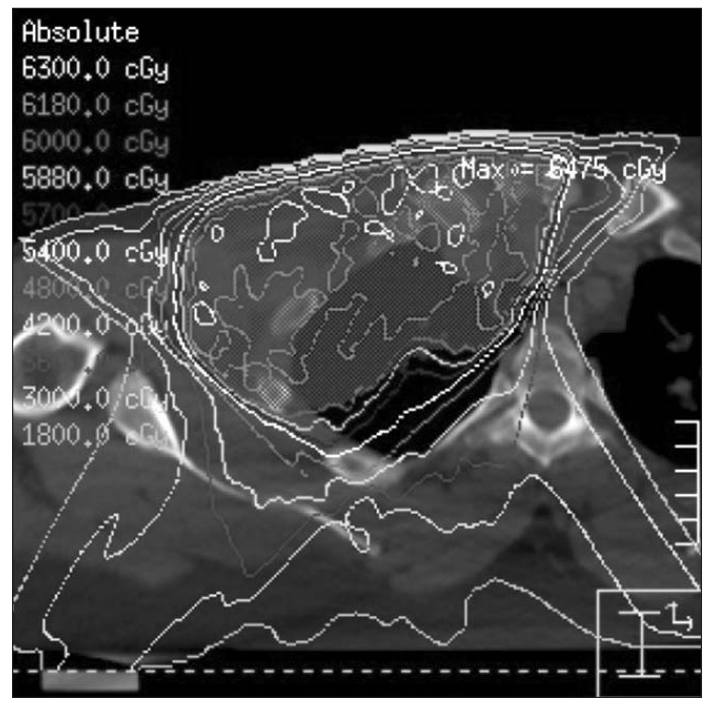

Fig. 1. Isodose lines from radiation plan.

for radiation therapy. Theoretically, these patients are already at risk of developing fibrosis from elevated TGF- $\beta$ and this can be further exacerbated by radiation exposure. It has been proposed that MFS patients requiring radiation therapy could be monitored throughout treatment via TGF- $\beta$ levels, specifically TGF- $\beta 1$, to determine the risk for fibrosis development [4]. However, only one case report exists discussing two MFS patients receiving radiation treatment and TGF- $\beta 1$ levels were not measured in these individuals [10]. Thus, it seems to be justified to explore a link between the risk for fibrosis development in MFS patients receiving radiation treatment and rising TGF- $\beta 1$ levels, as a tool to predict fibrosis in these patients.

\section{Case Report}

At age 31, an African-American male with MFS was diagnosed with a malignant peripheral nerve sheath tumor of the right brachial plexus. He carries a clinical diagnosis of MFS based on physical attributes in addition to a diagnosis of a type $\mathrm{A}$ aortic root dissection requiring aortic valve replacement. He subsequently developed worsening of cardiac failure which eventually led to a cardiac transplant. One year later, he presented with an increasing mass in the right supraclavicular region. He underwent a subtotal excision of the mass and final pathology demonstrated a malignant spindle cell neoplasm compatible with a malignant peripheral nerve sheath tumor. In the post-operative setting, he stated no changes in range of motion, no evidence of fibrosis, and no sensory or motor

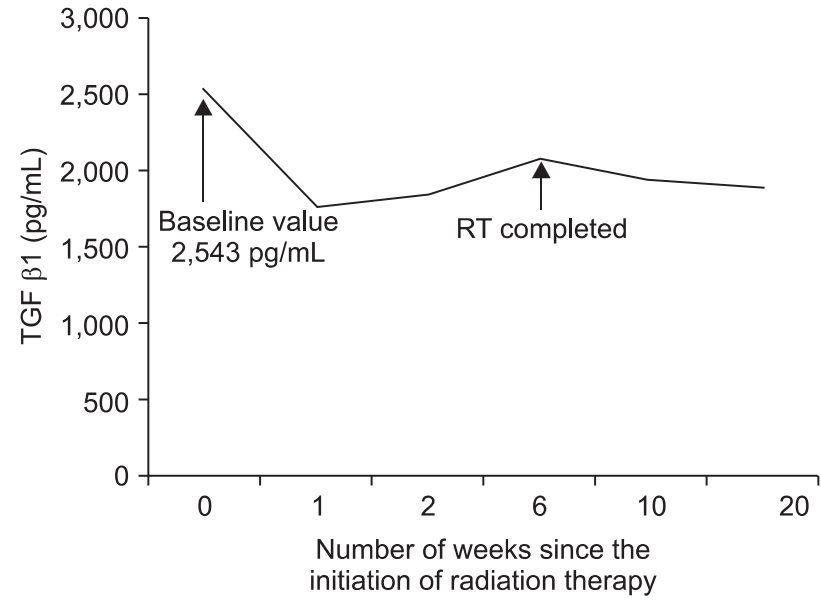

Fig. 2. TGF- $\beta 1(\mathrm{pg} / \mathrm{mL})$ values during and after radiation therapy. Baseline value of $2,543 \mathrm{pg} / \mathrm{mL}$ depicted in red.

deficits of the upper right extremity. The residual tumor and tumor bed was treated with an intensity modulated radiation therapy plan to a dose of $60 \mathrm{~Gy}$, utilizing a 7 field plan completed in 30 fractions (Fig. 1). The clinical target volume encompassed the gross residual tumor and operative bed. To create the planning target volume a $3-\mathrm{cm}$ expansion on the clinical target volume was used, creating a target volume of $984.8 \mathrm{~mL}$. A baseline TGF- $\beta 1$ level of $2,543 \mathrm{pg} / \mathrm{mL}$ was obtained using a quantitative multiplex bead assay method. This patient's baseline level was considered elevated when compared to normal individuals in some studies [4] but it is still within the normal range per laboratory standards.

During the course of radiotherapy, he experienced skin hyperpigmentation (RTOG grade 1) and dry cough (RTOG grade 1). Additional TGF- $\beta 1$ values during his treatment demonstrated no increase from baseline (Fig. 2). He completed all treatments without significant interruption. At one month follow-up from the completion of radiation, he presented with mild skin irritation and reported resolution of the dry cough. A repeat TGF- $\beta 1$ measurement was obtained at this visit which again showed no significant change from his baseline. He first reported fullness and decreased range of motion of his right upper extremity at the 5-month follow-up visit which persisted at his one year follow-up visit. He experienced difficulty with abduction of the shoulder beyond $120^{\circ}$, but it did not significantly impair his activities of daily living. MRI of the supraclavicular region revealed no evidence of recurrence but did demonstrate fibrosis in the surgical bed (Fig. 3). CT of the chest was also obtained which demonstrated no evidence of metastasis. However it showed radiation-induced changes 


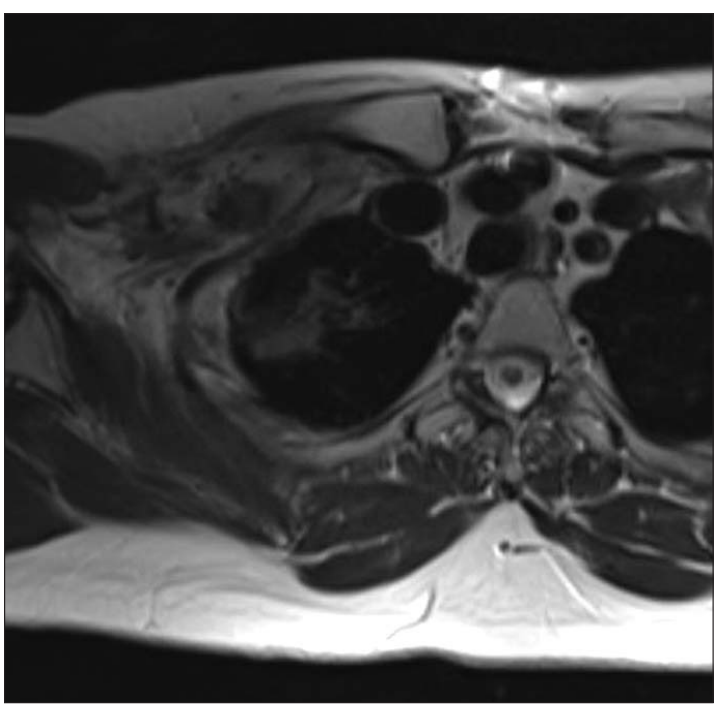

Fig. 3. Fibrosis in the right supraclavicular region.

of the right upper lobe correlating with the $60 \mathrm{~Gy}$ isodose line (Fig. 4). The patient continued to report no pulmonary symptoms after completion of therapy.

\section{Discussion}

In patients with collagen vascular disorders, such as MFS, there are limited tools available to determine the risk and extent of fibrosis that may develop secondarily to radiation therapy. Despite the development of several animal models designed to study MFS [12], no studies have investigated the potential risk of increased fibrosis after radiotherapy in this high-risk group. In one mouse model deficient for $F B N-1$, there was increased activation of TGF- $\beta$ and excess fibrosis $[5,6]$; however, these mice were not exposed to radiation.

Radiotherapy alone also has been shown to induce TGF- $\beta$ [7] by activating a TGF- $\beta$-specific transcription factor [13]. These effects have been noted in multiple regions of the body including the thorax, abdomen, and pelvis $[7,9-11,14,15]$. Due to these findings, patients with collagen vascular disease, such as MFS, are thought to be at an increased risk for fibrosis after treatment with radiation [16]. In fact, despite limited literature investigating the outcomes of patients with collagen vascular disease and radiation treatment, collagen vascular disease has been cited as a relative contraindication for radiation therapy [17].

This case report discussed herein, to our knowledge, is only the third published case of a MFS patient exposed to radiation.

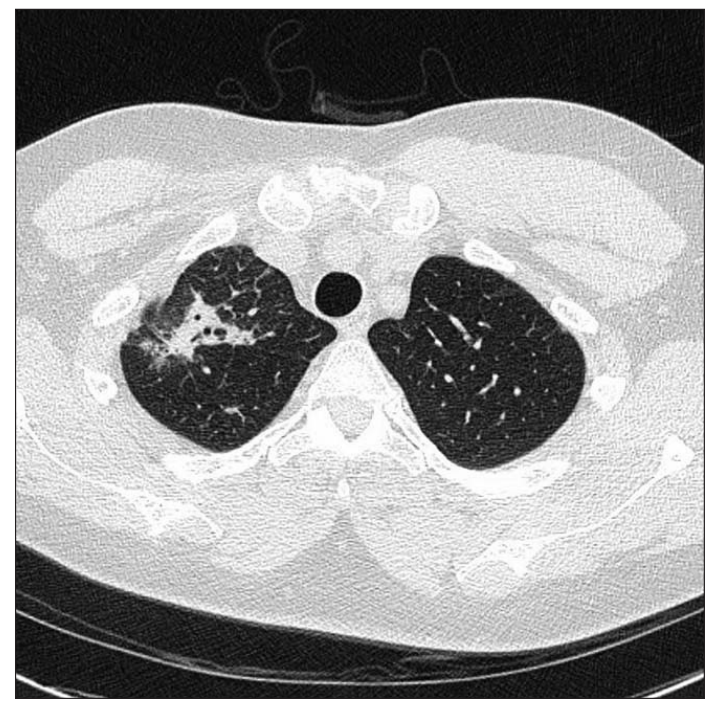

Fig. 4. Radiation fibrosis 5 months after completion of radiation therapy.

The first case report presented two patients who tolerated cranial or pelvic radiotherapy [4]. The authors suggested that TGF- $\beta 1$ levels could be used to monitor patients [4] as its level has been shown to correlate with radiation fibrosis in normal individuals $[4,9,11]$. In our report, we present a patient with MFS who was treated to $60 \mathrm{~Gy}$ to the right supraclavicular region while his TGF- $\beta 1$ levels were monitored. The initial TGF- $\beta 1$ level of $2,543 \mathrm{pg} / \mathrm{mL}(2.543 \mathrm{ng} / \mathrm{mL})$ measured in this patient is less than the previously reported mean level of 15 $\pm 1.7 \mathrm{ng} / \mathrm{mL}$ observed in MFS patients not on cardiovascular drugs and is closer to the normal control value of $2.5 \pm 0.4$ $\mathrm{ng} / \mathrm{mL}$ in that study [18]. One would anticipate that a patient with MFS undergoing radiation therapy would demonstrate a significant elevation in plasma TGF- $\beta 1$, yet that was not observed. Our patient's TGF- $\beta 1$ never increased from his baseline value, but he still developed clinically significant fibrosis of the supraclavicular region resulting in fullness and firmness of the area and decreased range of motion of the shoulder joint. Although these clinical findings are confounded by the fibrosis that can occur after surgery in this population; he also developed unexpected lung changes of the right upper lobe on CT imaging that was included in the radiation treatment field. Thus, this case report suggests that TGF- $\beta 1$ monitoring alone is not adequate to predict fibrosis outcomes for MFS patients undergoing radiation treatment.

The lack of change in TGF- $\beta 1$ after radiation exposure in our patient when compared to patients without collagen vascular 
disease is likely multifactorial. Most studies in non-MFS patients undergoing radiation treatment that demonstrated a correlation with pre- and/or post-treatment TGF- $\beta 1$ and fibrosis included patient who were treated with larger radiation fields [11]. In our patient, only the supraclavicular region with a small portion of the right upper lobe was irradiated. The increase of serum TGF- $\beta 1$ is dose and volume dependent and may therefore not be predictive when lower doses or smaller volumes are utilized. In cases where smaller volumes are irradiated, changes in TGF- $\beta 1$ can be seen on the molecular level via tissue samples $[7,19]$ rather than plasma levels. Unfortunately, tissue samples were not obtained in our patient to asses a local increase in TGF- $\beta 1$.

The histology of normal tissue being irradiated may also account for changes in detectable differences in plasma TGF- $\beta 1$. Most studies that detected a change in TGF- $\beta 1$ were for patients being treated for lung cancer. The alveolar macrophages and bronchial epithelial cells appear to be one source of TGF- $\beta 1$ in these patients [11]. The quantity of TGF- $\beta 1$ produced by other tissues is unknown and therefore may result in an unpredictable change in plasma TGF- $\beta 1$.

This clinical scenario also presents another potential application of the recently developed oral TGF- $\beta$ inhibitors. These agents cause disruption of the TGF- $\beta$ signaling pathway via small molecular receptor kinase inhibitors [19]. Initial studies in animal models suggest that the severity of radiation-induced lung injury in Sprague-Daley rats can be reduced with these inhibitors [16]. Also, losartan, an angiotensin II type 1 receptor blocker (ARB) that inhibits TGF- $\beta$ expression and activation, may provide another means of inhibiting fibrosis development in MFS patients and thus, allow their treatment with RT. This utility of losartan in patients with MFS was recently demonstrated in a pediatric study where patients with severe MFS treated with losartan alone or with a beta-blocker experienced a reduction in the change of aortic root diameter as compared to those only on a beta-blocker [20]. The application of losartan in the adult population is currently being investigated. Further development of these inhibitors will be particularly beneficial for patients with collagen vascular diseases, such as MFS patients, to reduce the extent of fibrosis while undergoing radiation therapy.

In conclusion, patients with collagen vascular disease who require radiation therapy pose a difficult clinical scenario as little is known regarding the extent of fibrosis induced by radiation in this high risk patient population. It has been proposed that monitoring TGF- $\beta 1$ levels in patients with MFS could be used to predict development of fibrosis. However, in our experience, TGF- $\beta 1$ was not predictive of radiation induced fibrosis as previously demonstrated in a normal patient population. We therefore caution against utilizing TGF- $\beta 1$ in patients with collagen vascular disorders to make treatment recommendations or to justify treating to a higher dose or larger volume. Further research will have to be done to determine if collagen vascular diseases truly are contraindication to radiation therapy, and if they are not, tests to better predict fibrosis development, perhaps TGF- $\beta 1$ levels via tissue samples, must be investigated.

\section{Conflict of Interest}

No potential conflict of interest relevant to this article was reported.

\section{References}

1. Cook JR, Nistala H, Ramirez F. Drug-based therapies for vascular disease in Marfan syndrome: from mouse models to human patients. Mt Sinai J Med 2010;77:366-73.

2. Robinson PN, Booms P, Katzke $S$, et al. Mutations of FBN1 and genotype-phenotype correlations in Marfan syndrome and related fibrillinopathies. Hum Mutat 2002;20:153-61.

3. Isogai $Z$, Ono RN, Ushiro $S$, et al. Latent transforming growth factor beta-binding protein 1 interacts with fibrillin and is a microfibril-associated protein. J Biol Chem 2003;278:2750-7.

4. Li C, Wilson PB, Levine E, Barber J, Stewart AL, Kumar S. TGFbeta1 levels in pre-treatment plasma identify breast cancer patients at risk of developing post-radiotherapy fibrosis. Int J Cancer 1999;84:155-9.

5. Martin M, Lefaix J, Delanian S. TGF-beta 1 and radiation fibrosis: a master switch and a specific therapeutic target? Int J Radiat Oncol Biol Phys 2000;47:277-90.

6. Neptune ER, Frischmeyer PA, Arking DE, et al. Dysregulation of TGF-beta activation contributes to pathogenesis in Marfan syndrome. Nat Genet 2003;33:407-11.

7. Hill RP, Rodemann HP, Hendry JH, Roberts SA, Anscher MS. Normal tissue radiobiology: from the laboratory to the clinic. Int J Radiat Oncol Biol Phys 2001;49:353-65.

8. Lafuma C, El Nabout RA, Crechet F, Hovnanian A, Martin M. Expression of 72-kDa gelatinase (MMP-2), collagenase (MMP1), and tissue metalloproteinase inhibitor (TIMP) in primary pig skin fibroblast cultures derived from radiation-induced skin fibrosis. J Invest Dermatol 1994;102:945-50.

9. Anscher MS, Murase T, Prescott DM, et al. Changes in plasma TGF beta levels during pulmonary radiotherapy as a predictor 
of the risk of developing radiation pneumonitis. Int J Radiat Oncol Biol Phys 1994;30:671-6.

10. Anscher MS, Peters WP, Reisenbichler $H$, Petros WP, Jirtle RL. Transforming growth factor beta as a predictor of liver and lung fibrosis after autologous bone marrow transplantation for advanced breast cancer. N Engl J Med 1993;328:1592-8.

11. Anscher MS, Kong FM, Andrews K, et al. Plasma transforming growth factor beta1 as a predictor of radiation pneumonitis. Int J Radiat Oncol Biol Phys 1998;41:1029-35.

12. Pereira L, Andrikopoulos K, Tian J, et al. Targetting of the gene encoding fibrillin-1 recapitulates the vascular aspect of Marfan syndrome. Nat Genet 1997;17:218-22.

13. Barcellos-Hoff MH, Derynck R, Tsang ML, Weatherbee JA. Transforming growth factor-beta activation in irradiated murine mammary gland. J Clin Invest 1994;93:892-9.

14. Anscher MS, Marks LB, Shafman TD, et al. Risk of long-term complications after TFG-beta1-guided very-high-dose thoracic radiotherapy. Int J Radiat Oncol Biol Phys 2003;56:988-95.

15. Nguyen NP, Antoine JE, Dutta S, Karlsson U, Sallah S. Current concepts in radiation enteritis and implications for future clinical trials. Cancer 2002;95:1151-63.

16. Phan C, Mindrum M, Silverman C, Paris K, Spanos W. Matched-control retrospective study of the acute and late complications in patients with collagen vascular diseases treated with radiation therapy. Cancer J 2003;9:461-6.

17. Winchester DP, Cox JD. Standards for diagnosis and management of invasive breast carcinoma. CA Cancer J Clin 1998;48:83-107.

18. Matt $P$, Schoenhoff $F$, Habashi J, et al. Circulating transforming growth factor-beta in Marfan syndrome. Circulation 2009;120:526-32.

19. Anscher MS, Thrasher B, Zgonjanin L, et al. Small molecular inhibitor of transforming growth factor-beta protects against development of radiation-induced lung injury. Int J Radiat Oncol Biol Phys 2008;71:829-37.

20. Brooke BS, Habashi JP, Judge DP, Patel N, Loeys B, Dietz HC 3rd. Angiotensin II blockade and aortic-root dilation in Marfan's syndrome. N Engl J Med 2008;358:2787-95. 